\title{
Ultrasonido en Obstetricia
}

\author{
Andrés Sarmiento*
}

\begin{abstract}
RESUMEN: Se revisa el ultrasonido como técnica diagnóstica dentro del contexto obstétrico moderno. Se enuncian aspectos de su clasificación, indicaciones, seguridad y uso rutinario. Se comenta acerca de las posibles implicaciones médico legales de la práctica sonográfica en la obstetricia actual.
\end{abstract}

PALABRAS CLAVES: Ultrasonido, clasificación, trimestre, médico-legal.

SUMMARY: Ultrasound is reviewed as a diagnostic technique in the context of modern obstetrics. Topics such as classification, indications, safety and routine use, are enunciated. Possible medico-legal implications of ultrasonographic practice in obstetrics are commented.

KEY WORDS: Ultrasonography, classification, trimester, legal aspects.

Desde su advenimiento a la obstetricia moderna, el ultrasonido se ha convertido en el medio diagnóstico más popular en la valoración del feto y del embarazo. Su inocuidad, fácil disponibilidad, caudal de información que aporta, bajo costo y ausencia de contraindicaciones, lo han convertido durante la última década en el procedimiento diagnóstico más valioso para el obstetra.

En la actualidad aproximadamente un $70 \%$ de la población obstétrica norteamericana es sometida a por lo menos un examen ecográfico durante su embarazo (1). En nuestro medio el ultrasonido diagnóstico se ha convertido en un examen de rutina dentro de la práctica obstétrica actual. Sin embargo, su uso rutinario y en ocasiones indiscriminado lleva al riesgo de convertirlo en un método diagnóstico de uso masivo y comercial; en ocasiones no existen conceptos claros en cuanto a sus verdaderas indicaciones, utilidad, momento de solicitud, seguridad y relación costo/beneficio. A esto se agrega que por parte del sonologista, pueden existir deficiencias en relación con un mínimo de información que debe suministrar.

En las siguientes líneas se revisará al ultrasonido como técnica diagnóstica; se analizarán los diferentes tipos de estudios, sus indicaciones y su utilidad. En general se informará al lector acerca del lugar que debe ocupar esta valiosa tecnología diagnóstica dentro del contexto obstétrico actual.

Médico Ginecobstetra. Coordinador Académico de la Unidad de Medicina Materno-Fetal. Escuela Colombiana de Medicina. Hospital Simón Bolívar. Bogotá, Director Científico OBGYN LTDA. Bogotá.

\section{Principios del ultrasonido diagnóstico}

El sonido puede ser representado como una onda con una velocidad (metros/sg), frecuencia (ciclos/sg) y longitud específicas. La frecuencia del sonido audible al oído humano oscila entre los 20-20000 ciclos/sg (hertz $\mathrm{o} \mathrm{Hz}$ ). Por lo tanto, el sonido con frecuencias mayores a 20000 ciclos/sg es clasificado como ultrasonido. Los aparatos utilizados en el ultrasonido diagnóstico utilizan frecuencias de 2-10 millones de hertz (2-10 megaHz $[\mathrm{MHz}])$. A mayor frecuencia de sonido menor es la profundidad de penetración diagnóstica, pero es mejor la resolución de la imagen. La gran mayoría de transductores transabdominales utilizan frecuencias de sonido de 3.5 - $5 \mathrm{MHz}$, mientras los transvaginales utilizan $5-7.5 \mathrm{MHz}$.

El ultrasonido diagnóstico hace uso de las características de ciertos cristales de vibrar y emitir ondas de sonido al recibir el estímulo de una corriente eléctrica, y de emitir una corriente eléctrica al ser estimulados con sonido (principio piezo eléctrico). El términotransductor se refiere pues, a un aparato capaz de convertir una forma de energía en otra; los transductores piezoeléctricos son elementos capaces de emitir y captar ondas ultrasónicas. El transductor de un ecógrafo emite ondas de sonido con una frecuencia específica; éstas al atravesar las interfases de tejido son reflejadas al emisor donde son transformadas a impulsos eléctricos que son representados como una señal visual en una pantalla. Este, es el principio del ultrasonido diagnóstico.

Durante el desarrollo del ultrasonido como técnica diagnóstica se han utilizado diversos tipos de técnicas; modo A, modo B, modo $\mathrm{M}$ y actualmente el ultrasonido de tiempo real y el doppler. 


\section{Seguridad del ultrasonido diagnóstico}

Desde el inicio de su utilización como medio diagnóstico, ha existido preocupación por la posibilidad que el ultrasonido implique riesgos sobre el feto. En 1988 el Instituto Americano de Ultrasonido en Medicina determinó $100 \mathrm{~mW} / \mathrm{cm}^{2}$ como el nivel máximo de seguridad para la exposición tisular al ultrasonido. Afortunadamente la gran mayoría de ecógrafos modernos no superan los $10-20 \mathrm{~mW} / \mathrm{cm}^{2}$, como energía determinada en la superficie del transductor (2).

Desde su uso como medio diagnóstico el ultrasonido no ha demostrado efectos biológicos nocivos sobre el operador, la paciente o el feto. La valoración pediátrica de pacientes expuestos durante la vida intrauterina al ultrasonido, no han demostrado alteraciones en lo que refiere a evaluación postparto, desarrollo neonatal, función cognoscitiva, función auditiva y visual, o déficit neurológico (3). Aún así, la recomendación universal sugiere el uso prudencial de métodos de ultrasonido en los cuales se utilice un poder acústico alto. En éstos se recomienda la menor exposición posible.

\section{Tipos de estudio}

El tipo de estudio solicitado debe variar en relación a la información que se busque. Una clasificación adecuada debe incluir qué tipo de estudio solicitar, cuándo solicitarlo y a qué pacientes.

\section{Clasificación: $(2,4)$}

A pesar que múltiples clasificaciones han sido propuestas para la práctica ultrasonográfica en obstetricia, la que universalmente viene siendo utilizada es aquella sugerida por el Colegio Americano de Obstetricia y Ginecología (ACOG). A esta clasificación deben someterse todos aquellos que practiquen el ultrasonido en obstetricia, independientemente de su nivel de entrenamiento.

Debe evitarse utilizar otras clasificaciones que son confusas tanto para el médico como para la paciente; imprimen ansiedad en la gestante y pueden caer en connotaciones comerciales.

I. Estudio obstétrico básico o de rutina

Está indicado en la valoración biométrica y biofísica fetal durante el seguimiento del embarazo. Si las condiciones técnicas y de edad gestacional lo permiten, debe incluir los siguientes parámetros:

- Confirmar embarazo.

- Número fetal

- Presentación y posición

- Viabilidad

- Valoración de líquido amniótico

- Localización y características placentarias

- Actividad biofísica fetal

- Valoración de masas pélvicas maternas

- Valoración anatómica fetal. Debe excluir malformaciones fetales mayores y debe valorar idealmente: SNC, espina, cuatro cavidades cardíacas y eje cardíaco, pared abdominal, presencia y normalidad de estómago, riñones y vejiga, extremidades, cordón y si es óptimamente visualizado, el sexo fetal.
Biometría fetal: debe contemplar por lo menos el análisis de cuatro parámetros biométricos básicos como son LF, CC, CA y DBP.

- Finalmente, un análisis somero por parte del operador que correlacione los hallazgos sonográficos con la indicación del estudio.

\section{Estudio obstétrico limitado}

Comprende el estudio de aspectos muy específicos del embarazo, sin comprender un análisis biométrico completo. Su solicitud puede resultar supremamente práctica pues evita la obtención de información innecesaria, limitándose a aspectos ecográficos muy concretos que son indispensables dentro del contexto de una situación clínica específica. Asimismo, disminuye costos para el paciente y tiempo para el ultrasonografista. Son ejemplos de ultrasonido limitado:

- Confirmación de presentación fetal

- Valoración de líquido amniótico (índice de L.A.)

- Perfil biofísico fetal

- Localización placentaria

- Guía ecográfica para amniocéntesis

- Confirmación de viabilidad fetal

III. Ultrasonido "comprensivo" o de detalle

Comprende un análisis biométrico y biofísico fetal muy completo haciendo énfasis en un exhaustivo recuento de la anatomía fetal. Está indicado en pacientes con embarazos de alto riesgo y específicamente en fetos con sospecha de malformaciones detectados en un US obstétrico de rutina, pacientes con exposición a teratógenos o en pacientes con antecedente de fetos malformados.

La práctica de este tipo de examen sonográfico debe realizarse entre las 16 y 28 semanas (idealmente 18-24 semanas) cuando la visualización de las partes fetales es óptima y el desarrollo anatómico fetal ha concluido. El examen debe ser practicado por un operador quien posea conocimientos de fisiología feto-materna y tenga la suficiente experiencia y entrenamiento para desarrollar este tipo de estudio e interpretar los hallazgos. El tiempo de examen toma por lo general 45 a 60 minutos.

En algunas ocasiones pueden existir fallas técnicas que no permitan la visualización de la totalidad de la anatomía fetal. Ejemplos de estas condiciones son obesidad materna, oligoamnios, un feto encajado y ocasionalmente una actitud fetal inadecuada.

En estos casos se prefiere reprogramar a la paciente.

Actualmente se utiliza la valoración anatómica fetal temprana a través del ultrasonido transvaginal $(5,8-9)$. Esta técnica permite obtener unos parámetros anatómicos fetales muy claros entre las 9 y 16 semanas de gestación. Nuevamente, su utilización requiere un operador experimentado con conocimientos de embriología y un equipo sonográfico actualizado. Su única indicación y además gran ventaja, radica en poder detectar tempranamente alteraciones anatómicas en fetos con factores plenamente establecidos de alto riesgo para desarrollar malformaciones.

\section{Ecografía obstétrica básica y la edad gestacional}

En cada trimestre los hallazgos sonográficos varían ampliamente y la ecografía obstétrica de rutina arrojará 
un tipo de información muy diferente de acuerdo a la edad gestacional.

* Primer trimestre: con los recursos tecnológicos disponibles en la actualidad, el examen sonográfico antes de las 12 semanas se prefiere realizar con sonda Transvaginal. Esta tecnología permite una muy clara visualización de las estructuras embrionarias y uterinas.

Una ecografía transvaginal de primer trimestre debe incluir la siguiente información:

- Localización del saco gestacional

Identificación de polo embrionario o fetal y su número

- Imágenes asociadas a viabilidad

- Edad gestacional aproximada (longitud cráneocaudal)

- Presencia de actividad cardíaca

- Descripción de otras estructuras embrionarias como el amnios, saco vitelino e implantación placentaria. Opcionalmente descripción del orificio cervical interno (OCI) y longitud endocervical.

- Valoración y seguimiento del desarrollo anatómico embrionario según la semana específica de embarazo.

- Descartar masas pélvicas maternas.

El saco gestacional puede ser visualizado por US transvaginal desde las 4 semanas de embarazo o 15-18 días postovulación. Se asocia a una zona discriminatoria para HCG promedio, mayor de $1000 \mathrm{mIU} / \mathrm{ml}$ (rango de 600$1025 \mathrm{mIU} / \mathrm{ml}$ ). (5) El saco vitelino se define desde las 5 semanas mientras el polo fetal se visualiza desde las 5-6 semanas. Después de la semana sexta se debe identificar actividad cardíaca embrionaria en la totalidad de casos.

La medición de la longitud cráneo-caudal entre las 8 y 13 semanas permite determinar la edad gestacional con una exactitud de más o menos 5 días $(2,8)$.

Las principales patologías asociadas a la gestación y que son las indicaciones más frecuentes de ultrasonido de primer trimestre son: el huevo anembrionado, la pérdida fetal temprana (aborto "retenido"), embarazo ectópico, embarazo múltiple y los sangrados del primer trimestre asociados a separación corio-amniótica o hematoma subcoriónico.

* Segundo trimestre: la práctica del estudio sonográfico antes de las 28 semanas permite una valoración de múltiples parámetros biométricos y la valoración de la anatomía fetal en una etapa donde la visualización es óptima. Quizás es la edad gestacional ideal para practicar el examen en casos donde la paciente únicamente tiene acceso a un solo estudio sonográfico durante toda su gestación.

El estudio inicialmente debe describir la presentación, posición y actitud fetal. La valoración biométrica como mínimo debe incluir longitud del fémur (LF), circunferencia abdominal (CA), circunferencia cefálica (CC) y diámetro biparietal (DBP). La medición de otras variables biométricos es válida y puede incluir húmero, tibia, diámetro binocular u orbital, longitud del pie y diámetro cerebelar, entre otros (6). El análisis biométrico debe correlacionarse con la fecha de la última menstruación (FUM) y/o con estudios sonográficos previos corroborando la edad gestacional y el adecuado desarrollo fetal. La manera más sencilla y rápida de correlacionar estudios sonográficos seriados tratando de establecer un adecuado crecimiento fetal, es comparar la fecha posible de parto (FPP) que se determine para cada estudio, entre sí; y con aquella que corresponda a la FUM. Dentro de la vigilancia del desarrollo fetal la estimación sistemática del peso fetal, es de suma importancia.

El estudio además debe incluir la descripción del aspecto y localización placentaria, la visualización y características del cordón umbilical y una cuantificación del volumen del líquido amniótico. La visualización óptima de la relación entre el borde placentario y el OCI en casos de pacientes "bajas", debe practicarse mediante US transvaginal. Finalmente, el operador debe adicionar una apreciación de la actividad biofísica fetal en relación con la edad gestacional establecida.

* Tercer trimestre: la valoración biométrica pierde objetividad durante el tercer trimestre; las variaciones en la determinación de la edad gestacional pueden ser del orden de \pm 3 semanas, aún en las mejores manos. Sin embargo, la evaluación biométrica juiciosa si debe permitir una valoración del crecimiento fetal.

La valoración de las relaciones antropométricas entre sí ( $\mathrm{LF} / \mathrm{CA}, \mathrm{CC} / \mathrm{CA})$ tiene un gran valor en el diagnóstico de las alteraciones del crecimiento fetal.

El cálculo del peso fetal a esta etapa del embarazo es de suma importancia. Con los métodos actuales la estimación del peso fetal y en manos experimentadas, no debe sobrepasar un $15 \%$ de certeza en relación con el peso neonatal (10). Desafortunadamente el cálculo del peso fetal es pobre en fetos macrosómicos o pequeños para la edad gestacional. El tercer trimestre no es el momento adecuado para practicar un análisis completo de la anatomía fetal; por lo general en esta etapa de la gestación la visualización fetal no es la mejor.

El análisis de la presentación, posición y actitud fetal son importantes para el parto. La descripción de las características de la placenta, el volumen del líquido amniótico y la actividad biofísica junto con el peso fetal, son los parámetros más importantes que busca el clínico al solicitar un estudio a esta edad gestacional.

\section{Aspectos médico-legales del ultrasonido}

Los problemas que por lo general llevan a litigios médico-legales en relación con estudios o a procedimientos asociados al diagnóstico sonográfico, son: (6)

- Equipo no actualizado

- Inadecuado entrenamiento del operador

- Juicio clínico

- Fallas diagnósticas de: malformaciones fetales, embarazo ectópico, viabilidad, placenta previa y embarazo múltiple.

- Complicación de procedimientos invasivos guiados por ultrasonido.

La época que vivimos obliga a que aquél que practique el diagnóstico sonográfico de alta resolución lo haga en equipos actualizados. El entrenamiento para un médico radiólogo o ginecólogo que pretenda practicar ultrasonido obstétrico básico debe incluir según el cri- 
terio de la Asociación Americana de Ultrasonido en Medicina, tres meses de entrenamiento supervisado por personal idóneo y por lo menos 200 estudios de experiencia institucional (6).

Un juicio clínico deficiente en especial para el operador con experiencia limitada, es motivo frecuente de demandas. La posibilidad de "segundas opiniones" o revaloraciones es una conducta prudente en casos difíciles. El operador siempre debe conocer sus limitaciones. Asimismo, es indispensable que el operador esté en capacidad de practicar una correlación clínicoimagenológica adecuada; ocasionalmente es él quien primero advierte condiciones patológicas que requieren intervención urgente por parte del clínico.

La incidencia global de malformaciones congénitas es del $2 \%$ y $15 \%$ para malformaciones mayores y menores, respectivamente. Se define malformación congénita mayor como aquélla que tiene importancia médica, quirúrgica o cosmética para el neonato; con impacto en su morbimortalidad. La etiología de las anomalías congénitas pueden identificarse hasta en un $40 \%$ de casos y habitualmente $(20 \%)$ se debe a origen multifactorial. Existe una alta frecuencia en la asociación de anomalías congénitas con anomalías cardíacas $(23 \%)$ y cromosomopatías $(30 \%)(11)$.

Las malformaciones fetales más frecuentemente asociadas a fallas diagnósticas son: labio leporino, hernia diafragmática, defectos cardíacos, displasias esqueléticas y lesiones de la espina $(2,4)$.

Desafortunadamente, la certeza diagnóstica del ultrasonido en la detección de anomalías congénitas no es la ideal. La sensibilidad y especificidad del ultrasonido en la detección de anomalías varía de acuerdo al nivel de experiencia del ultrasonografista. La bibliografía disponible refiere una sensibilidad del ultrasonido para la detección de anomalías congénitas del 16-84\%, con especificidad del 91-100\% (11).

Finalmente, toda paciente que se someta a un procedimiento invasivo diagnóstico o terapéutico que implique aún un mínimo riesgo para el embarazo, debe ser advertida en conjunto con su pareja de estos riesgos y posibles complicaciones. En el contexto de la medicina "defensiva" que practicamos en la actualidad, es la paciente antes de iniciar el procedimiento.

\section{Uso rutinario del ultrasonido}

Aún cuando el uso rutinario del ultrasonido en población obstétrica general no ha demostrado que mejore el resultado perinatal, sí se han determinado unas indicaciones muy precisas que justifican su solicitud. En población obstétrica de bajo riesgo es conveniente analizar la relación costo/beneficio antes de decidir si toda gestante debe ser llevada a una sonografía. Estudios como el "RADIUS" que hace unos años pretendieron demeritar al ultrasonido como práctica rutinaria dentro de la obstetricia moderna han sido desmentidos por estudios multicéntricos más recientes que han demostrado beneficios en términos de morbimortalidad neonatal (11).

\section{Conclusión}

El ultrasonido es la técnica diagnóstica más valiosa con la que actualmente dispone el obstetra. A pesar de su gran popularidad, su uso debe ser racional y plegado a unas indicaciones muy precisas. El clínico debe conocer qué tipo de información busca al solicitar el estudio. Los estudios que no sean "básicos o de rutina", deben ser practicados por personal idóneo en la materia; idealmente obstetras con entrenamiento en medicina maternofetal.

\section{BIBLIOGRAFIA}

1. Horger ED III, Tsai CC. Ultrasound and the prenatal diagnosis congenital anomalies: a medico legal perspective. Obstet. Gynecol. 1989; 74: 617-619.

2. ACOG Technical Bulletin. Uttrasonography in Pregnancy. Número 187, diciembre de 1993.

3. Stark CR, Orleans M, Haverkamp AD, Murphy J. Short and long-term risksafter exposureto diagnostic ultrasound in utero. Obstet Gynecol 1984; 63: 194-200.

4. ACOG committee opinion. Ultrasound Imaging in Pregnancy. Committee in obstetrics: Maternal and Fetal Medicine. Número 96, Agosto 1991.

5. Timor Trisch I, Rotten S. Normal and abnormal fetal anatomy in the first 15 weeks of pregnancy using transvaginal ultrasound, p. 353380. Diagnostic ultrasound applied to Obstetrics and Gynecology. Sabbagar. Lippincott Co, $3^{\text {a }}$ edicion, 1995.

6. Ott, W. Clinical Obstetric Ultrasound. Obstetrical Ultrasound Manual, p 2-25. StJohn's Mercy Medical Center, St Louis, Mo 1989.
7. Shapiro B, Escobar M, Macuch R, Lavy G, De Cherney AH. A modelbased prediction for transvaginal ultrasonographic identification of early intrauterine pregnancy. Am. J. Obstet. Gynecol. 1992; 166: $1495-1500$.

8. Timor-Tritsch IE, Farine D, Rosen MG. A close look at early embrionic development with the high-frequency transvaginal transducer. Am. J. Obstet. Gynecol. 1988; 159: 676-681.

9. Cullen MT, Green J, Whetham J, Salafia C, Gabrielli S, Hobbins JC. Transvaginal Ultrasonographic detection of congenital anomalies in the first trimester. Am. J. Obstet. Gynecol. 1990; 163: 466-476.

10. Hadlock F. Sonographic estimation of Fetal age and Weight. Fetal ultrasound. Hadlock F. p. 39-48. The Radiologic Clinics of North America. 1990.

11. Romero R. Prenatal Diagnosis of Congenital Anomalies. En: Sonography in Obstetrics and Gynecology. Fleischer A, Boston; Appleton Lange; $5^{\text {a }}$ edition 1996; 543-575. 